\title{
Test of the proximity theorem for deformed nuclei
}

\author{
M. Seiwert and W. Greiner \\ Institut für Theoretische Physik der Universität Frankfurt am Main, Frankfurt am Main, Federal Republic of Germany \\ V. Oberacker \\ Department of Physics and Astronomy, Vanderbilt University, Nashville, Tennessee 37235
}

M. J. Rhoades-Brown

Physics Division, Oak Ridge National Laboratory, Oak Ridge, Tennessee 37830

(Received 3 June 1983)

\begin{abstract}
We compare a proximity-type potential for two interacting nuclei with the double-folding method. Both spherical and deformed systems are considered. Special "orientation windows" are found for two deformed nuclei giving rise to nuclear cohesion. If the same nucleon-nucleon interaction is utilized, the proximity and the double-folding potentials agree fairly well for a spherical + deformed system. However, deviations are found in the case of two deformed nuclei.
\end{abstract}

\section{INTRODUCTION}

As proposed by Bass ${ }^{1}$ and by Blocki et al., ${ }^{2}$ the interaction potential $V$ between two nuclei can be approximated by an expression of the form

$$
V_{N}(s)=F(\text { geometry }) \phi(s) \text {. }
$$

The dependence upon the surface separation $s$ is completely determined by the function $\phi(s)$; the shapes and relative orientations of both nuclei are described by a geometrical factor $F$. This approximation, called the "proximity theorem," can be understood in the following way: Using nonrelativistic many-body theory it is straightforward to derive the double-folding potential

$$
V_{N}=\int d V_{1} \int d V_{2} \rho_{1}\left(\overrightarrow{\mathrm{r}}_{1}\right) v\left(\overrightarrow{\mathrm{r}}_{12}, \ldots\right) \rho_{2}\left(\overrightarrow{\mathrm{r}}_{2}\right) .
$$

Equation (2) holds for small overlaps of the nuclear density distribution where exchange effects (Pauli principle) can be neglected. The effective nucleon-nucleon interaction $v$ depends upon the relative distance $\overrightarrow{\mathrm{r}}_{12}$ and momentum $\overrightarrow{\mathrm{p}}_{12}$ of the nucleons, as well as their spins and isospins.

Let us suppose that the distance $s$ between the two nuclear surfaces (see Fig. 1) is small (on the order of a few $\mathrm{fm})$. Due to the short-range character of $v\left(\overrightarrow{\mathrm{r}}_{12} \cdots\right)$ the interaction will be confined to a small region in space characterized by the volume elements $V_{1}$ and $V_{2}$. If we assume these volume elements to be infinitesimal, for simplicity, Eq. (2) reduces to

$$
\begin{aligned}
V_{N} & =d V_{1} \rho_{1}\left(\overrightarrow{\mathrm{r}}_{1}\right) d V_{2} \rho_{2}\left(\overrightarrow{\mathrm{r}}_{2}\right) v\left(\overrightarrow{\mathrm{r}}_{12}, \ldots\right) \\
& =d V_{1} \rho\left(\overrightarrow{\mathrm{R}}_{1}\left(\Omega_{1}^{M}\right)\right) d V_{2} \rho\left(\overrightarrow{\mathrm{R}}\left(\Omega_{2}^{M}\right)\right) v(\overrightarrow{\mathrm{s}}, \ldots) .
\end{aligned}
$$

The size of the interacting volume elements $d V_{i}$ depends on the range of the interaction and on the radii of curvature of the corresponding surface areas. Therefore, Eq. (3) reduces to (1) for given density distributions. Up to now, the proximity potential has been derived for two spherical $^{2}$ and for one spherical and one deformed nucleus. $^{3}$ In Sec. II, we carry out the above ideas to describe the nuclear interaction of two deformed nuclei. Our derivation is quite different from that given in a recent publication, ${ }^{4}$ but the final formulae are equivalent. We give here the first application of the proximity potential for two deformed nuclei. In principle, the proximity theorem should hold for an arbitrary short-range interaction. We shall solve the double-folding integrals numerically for various nucleon-nucleon potentials and compare the exact results for spherical and deformed systems with the proximity prescription. Limitations of the latter are discussed.

\section{PROXIMITY POTENTIAL FOR TWO DEFORMED NUCLEI AND COMPARISON WITH THE DOUBLE FOLDING METHOD}

The deformations of the two nuclei will be expressed in terms of the collective surface variables

$$
R_{i}\left(\Omega_{i}\right)=R_{0 i}\left[1+\sum_{\lambda \mu} \alpha_{\lambda \mu}^{(i)} Y_{\lambda \mu}^{*}\left(\Omega_{i}\right)\right], i=1,2,
$$

where the $z$ axes of the laboratory systems of nucleus 1 and 2 are chosen in the direction of the internuclear distance $\overrightarrow{\mathrm{r}}$. The coordinate axes $x_{i}$ and $y_{i}$ are parallel to each other. This is illustrated in Fig. 2.

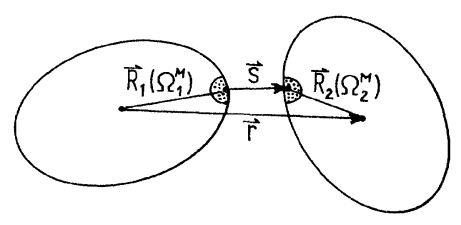

FIG. 1. Two nuclei with surface separation $s$ and interacting volume parts. 


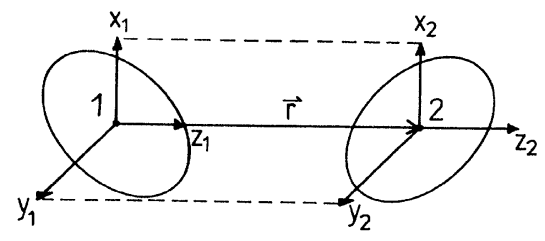

FIG. 2. Choice of the laboratory systems.

By a transformation to the intrinsic frames of reference, we can separate the deformation and orientation degrees of freedom

$$
a_{\lambda \nu}^{(i)}=\sum_{\mu} D_{\mu \nu}^{\lambda *}\left(\alpha_{i}, \beta_{i}, \gamma_{i}\right) \alpha_{\lambda \mu}^{(i)} .
$$

The Euler angles specify the relative orientation of the nuclei with respect to the two-center distance $\vec{r}$, and the tensors $a_{\lambda v}$ denote the intrinsic deformations. The problem can be simplified considerably if the following conditions are fulfilled:

(i) The intrinsic nuclear shapes are axially symmetric; in this case, the potential is independent of the Euler angles $\gamma_{i}$.

(ii) The body-fixed symmetry axes $z_{1}^{\prime}$ and $z_{2}^{\prime}$ are in the same plane (see Fig. 3). Hence, the problem does not depend anymore on $\alpha_{i}$.

The interaction potential between the two nuclei is now a function of three collective coordinates only: $V_{N}=V_{N}\left(r, \beta_{1}, \beta_{2}\right)$. Making use of the above approximations, we can write (see Fig. 1)

$$
\begin{aligned}
s & =\min _{\mathbf{\Omega}_{1}, \mathbf{\Omega}_{2}}\left|\overrightarrow{\mathbf{r}}+\overrightarrow{\mathbf{R}}_{2}\left(\Omega_{2}\right)-\overrightarrow{\mathbf{R}}_{1}\left(\Omega_{1}\right)\right| \\
& =\left|\overrightarrow{\mathbf{r}}+\overrightarrow{\mathbf{R}}_{2}\left(\Omega_{2}^{M}\right)-\overrightarrow{\mathbf{R}}_{1}\left(\Omega_{1}^{M}\right)\right| .
\end{aligned}
$$

The minimum distance between the two nuclear surfaces, specified by the angles $\Omega_{i}^{M}$, is determined by a numerical iteration procedure. The iteration must be carried out separately for any given set of deformation and orientation variables and for every internuclear distance $r$.

In Ref. 2 the geometrical factor $F$ was determined for a gap between two elliptic paraboloids with tip distance $s$, with radii of curvature $P_{i}$ and $\rho_{i}$ in the principal planes of curvature through the tip of paraboloid $i$, and an azimuthal angle $\phi$ between the principal planes of curvature of 1 and 2 (see Fig. 4):

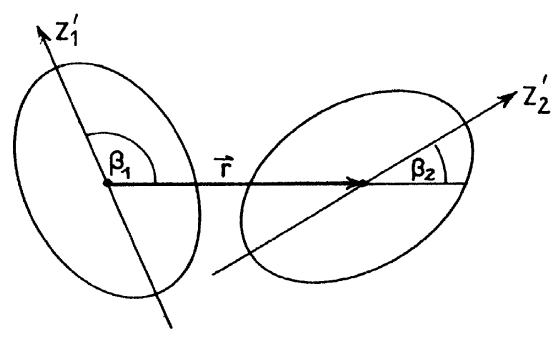

FIG. 3. Definition of the orientation angles $\beta_{1}, \beta_{2}$.

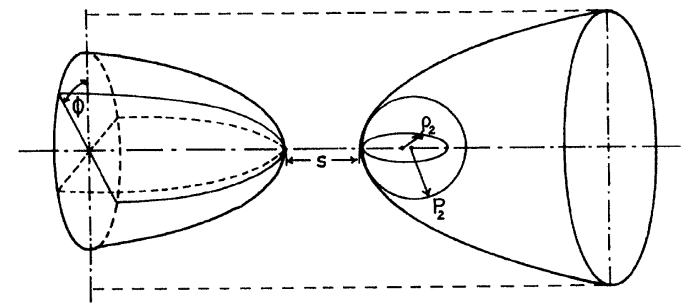

FIG. 4. Geometrical properties of the two paraboloids.

$$
\begin{aligned}
& F=4 \pi \gamma\left[\frac{1}{P_{1} \rho_{1}}+\frac{1}{P_{2} \rho_{2}}+\left[\frac{1}{P_{1} P_{2}}+\frac{1}{\rho_{1} \rho_{2}}\right] \sin ^{2} \phi\right. \\
& \left.+\left[\frac{1}{P_{1} \rho_{2}}+\frac{1}{P_{2} \rho_{1}}\right] \cos ^{2} \phi\right]^{-1 / 2}, \\
& \gamma=0.9517\left[1-1.7826\left(\frac{N-Z}{A}\right)^{2}\right] \mathrm{MeV} / \mathrm{fm} .
\end{aligned}
$$

With this ansatz, the nuclear surface is well reproduced near the $s$ axis, where the interacting volume elements $d V_{1}$ and $d V_{2}$ are located.

Due to the short-range character of the nuclear force, it is assumed that only these two infinitesimal volume elements contribute to the integral, Eq. (2). To a good approximation, the nuclear shapes may be replaced by paraboloids in the neighborhood of the distance vector $\vec{s}$. The radii of curvature must be taken at the surface points specified by the angles $\Omega_{i}^{M}$ and in the direction of their tangential plane. Due to the requirement that $s$ be the minimum distance between the nuclear surfaces,

$$
\frac{+\overrightarrow{\mathrm{s}}}{|s|} \text { and } \frac{-\overrightarrow{\mathrm{s}}}{|s|}
$$

are the normal vectors of the tangential plane of nuclei 1 and 2, respectively.

For axially symmetric nuclei, the principal radii of curvature are in the direction of the intrinsic unit vectors $\vec{e}_{\vartheta}$ and $\overrightarrow{\mathrm{e}}_{\varphi}$ (see Fig. 5). An arbitrary plane through the vector $\vec{e}_{\vartheta}$ includes the center of mass (see Figs. 3 and 4). There-

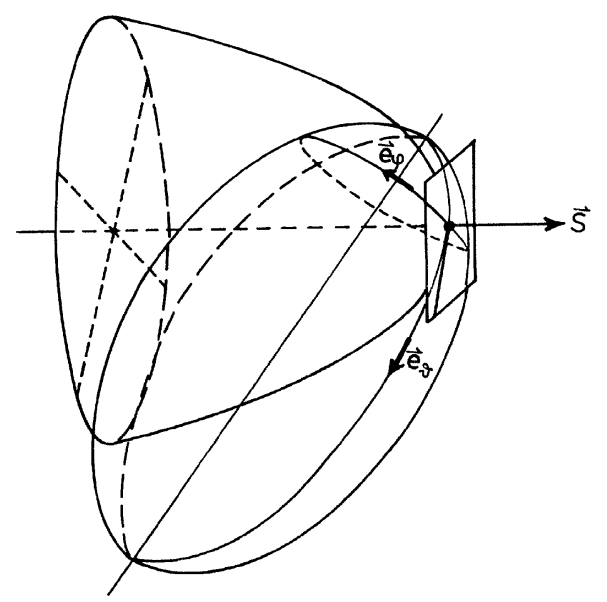

FIG. 5. Approximation of the nuclear shape by a coaxial elliptic paraboloid. The tangential plane is parallel for both surfaces. 


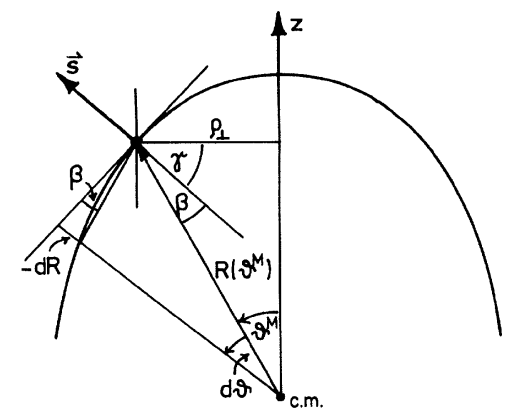

FIG. 6. Geometrical properties for the calculation of $\rho$.

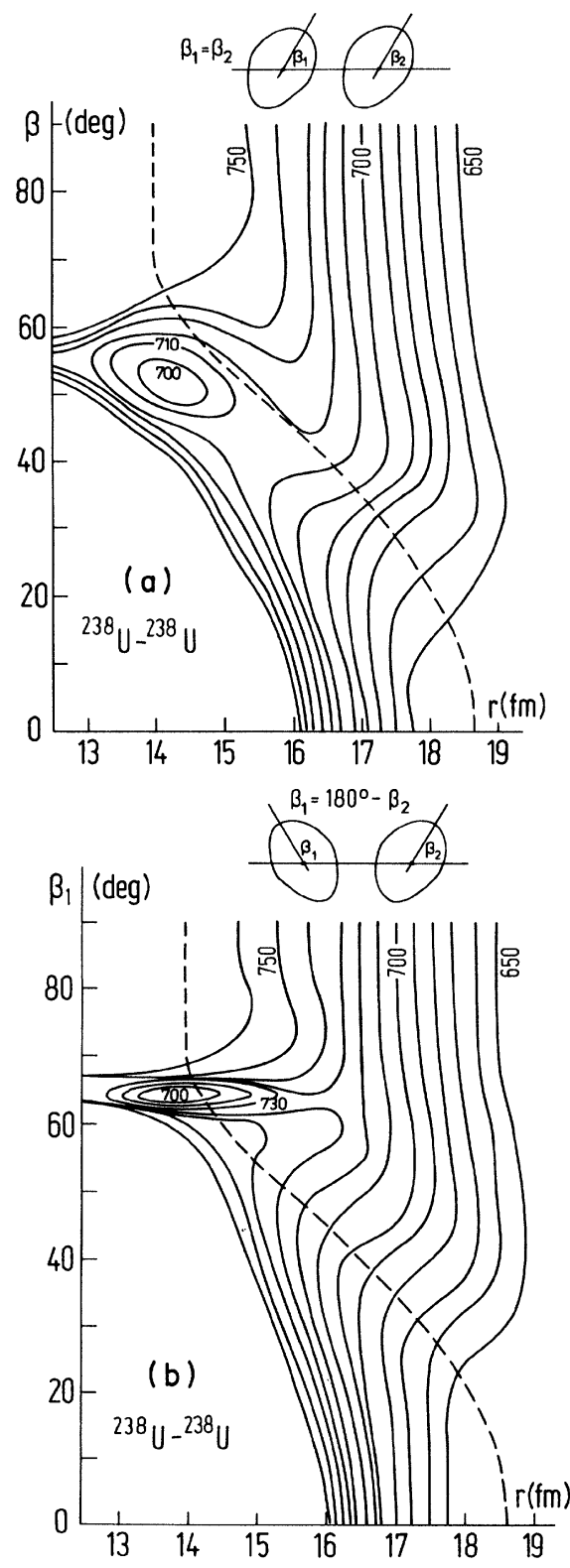

FIG. 7. Nuclear potential of two ${ }^{238} \mathrm{U}$ nuclei $\left(\delta_{2}=0.264, \delta_{4}=0.106\right)$ as function of the two center distance $r$ and the orientation angles $\beta_{1}$ and $\beta_{2}$ in the proximity approximation. The dashed line indicates the touching point of the two surfaces. fore the radius of curvature $P$ along $\overrightarrow{\mathrm{e}}_{\boldsymbol{\vartheta}}$ is simply given by

$$
P\left(\vartheta^{M}\right)=\frac{\left[R^{2}\left(\vartheta^{M}\right)+R^{\prime 2}\left(\vartheta^{M}\right)\right]^{3 / 2}}{R^{2}+2 R^{\prime 2}-2 R R^{\prime \prime}} \quad\left(R^{\prime}=\frac{\partial R}{\partial \vartheta}\right)
$$

The derivation of the radius of curvature $\rho$ along $\overrightarrow{\mathrm{e}}_{\varphi}$ is somewhat involved (see Fig. 6). We first define $\rho_{\perp}$ as the radius of curvature with the principal plane parallel to the intrinsic $z$ axis.

$$
\rho_{\perp}=R\left(\vartheta^{M}\right) \sin \vartheta^{M} .
$$

The theorem of Meusnier yields a connection between the radii of curvature of curves through the same point but in connection to different planes, in our case:

$$
\rho_{1}=\rho \cos \gamma,
$$

where $\gamma$ is the angle between the normal vector and $\rho_{\perp}$. The angle $\beta$ between $R\left(\vartheta^{M}\right)$ and the normal vector is easily calculated to be

$$
\tan \beta=\frac{-d R}{d \vartheta R}=-\frac{R^{\prime}}{R} .
$$

Summarizing the results of Eqs. (10)—(12), we finally obtain

$$
\rho\left(\vartheta^{M}\right)=\frac{R\left(\vartheta^{M}\right) \sin \vartheta^{M}}{\cos \left[\frac{\pi}{2}-\vartheta^{M}-\arctan \left(-\frac{R^{\prime}}{R}\right)\right]}
$$

This formula holds for $\vartheta^{M} \neq 0$. It is easy to see that $P\left(\vartheta^{M}=0\right) \equiv \rho\left(\vartheta^{M}=0\right)$. Equations (7), (9), and (13) determine the geometrical factor $F$ completely.

In the original paper (Ref. 2), the proximity function $\phi(s)$ was calculated in the Thomas-Fermi approximation using the Seyler-Blanchard N-N interaction ${ }^{5}$ :

$$
\begin{aligned}
& v\left(r_{12}, P_{12}\right)=-328.61 \mathrm{MeV} \frac{e^{-r_{12} / a}}{r_{12} / a}\left[1-\frac{P_{12}^{2}}{b^{2}}\right), \\
& a=0.62567 \mathrm{fm}, \quad b=392.48 \mathrm{MeV} / c .
\end{aligned}
$$

This phenomenological interaction is of a Yukawa type; the momentum dependent term simulates compression effects in the overlap region. The resulting heavy-ion potential function $\phi(s)$ [see Eq. (1)] is given by the "pocket formula":

$$
\begin{array}{r}
\phi(s \leq 1.2511 \mathrm{fm})=-\frac{1}{2}\left(s-s_{0}\right)^{2}-0.0852\left(s-s_{0}\right)^{3}, \\
s_{0}=2.54 \mathrm{fm} \\
\phi(s \geq 1.2511 \mathrm{fm})=-3.437 \exp (-s \mid 0.75 \mathrm{fm}) .
\end{array}
$$

Negative $s$ values correspond to the overlap region. It must be mentioned, however, that in this case the definitions of $s$ and $\Omega_{i}^{M}$ become somewhat arbitrary. Therefore, we choose the direction of $s$ to be parallel to $\overrightarrow{\mathrm{r}}$ in the overlap regions. With increasing overlap, the proximity model becomes more and more inaccurate since the interacting volume elements do not remain small. In this case, the description of the potential in terms of a single distance coordinate $s$ between the surface elements breaks down. In addition to the nuclear heavy-ion potential, we have to determine the Coulomb interaction 


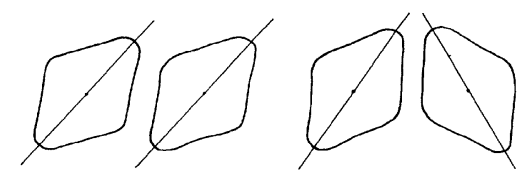

FIG. 8. Flat surface areas facing each other for two special orientations (schematic).

$V_{C}(\overrightarrow{\mathrm{r}})=\int d^{3} r_{1} \int d^{3} r_{2} \rho_{1}^{(P)}\left(\overrightarrow{\mathrm{r}}_{1}\right)\left|\overrightarrow{\mathrm{r}}+\overrightarrow{\mathrm{r}}_{2}-\overrightarrow{\mathrm{r}}_{1}\right|^{-1} \rho_{2}^{(P)}\left(\overrightarrow{\mathrm{r}}_{2}\right)$

The double-folding integral (16) can be evaluated numerically for two arbitrary charge distributions $\rho^{(P)}$ using several Fourier transformations and multipole expansions. If the Fourier transform of a function $f(\vec{x})$ is denoted by $\widetilde{f}(\overrightarrow{\mathrm{k}})$, we find

$V_{C}(\overrightarrow{\mathrm{r}})=(2 \pi)^{-3} \int d^{3} k \exp (-i \overrightarrow{\mathrm{k}} \cdot \overrightarrow{\mathrm{r}}) \frac{4 \pi}{k^{2}} \widetilde{\rho}_{1}^{(P)}(k) \widetilde{\rho}_{2}^{(P)}(-k)$.

Expanding all "plane waves" $\exp (i \overrightarrow{\mathrm{k}} \cdot \overrightarrow{\mathbf{R}})$ into their multipole components

$$
\exp (i \overrightarrow{\mathrm{k}} \cdot \overrightarrow{\mathbf{R}})=4 \pi \sum_{L, M} i^{L} j_{L}(k R) Y_{L M}(\hat{k}) Y_{L M}^{*}(\hat{R}),
$$

a general formula has been derived which involves only one-dimensional integrals. It is then possible to calculate the Coulomb interaction potential numerically to an accu-

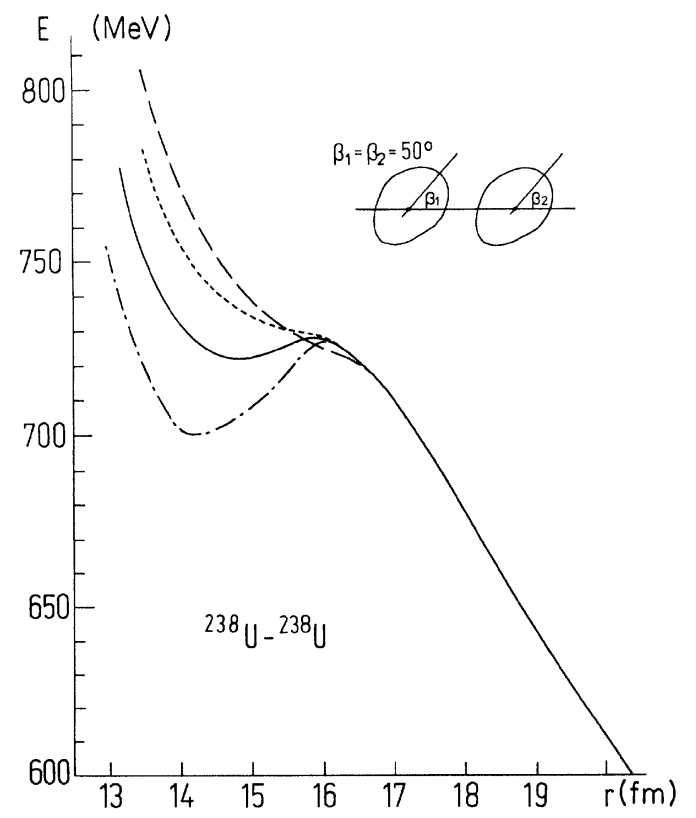

FIG. 9. The dependence of the nuclear potential on the hexadecupole deformation as a function of $r$ for the orientation angles $\beta_{1}=\beta_{2}=50^{\circ}$. The deformation parameters are $\delta_{2}=0.277$, $\delta_{4}=0.013$ (dashed line) from myonic $x$ rays (Ref. 8), $\delta_{2}=0.226$, $\delta_{4}=0.052$ (dotted line) from proton scattering (Ref. 9); $\delta_{2}=0.261, \delta_{4}=0.087$ (solid line) from electron scattering (Ref. 10); and $\delta_{2}=0.261, \delta_{4}=0.106$ (dashed-dotted line) from Coulomb excitation (Ref. 7).

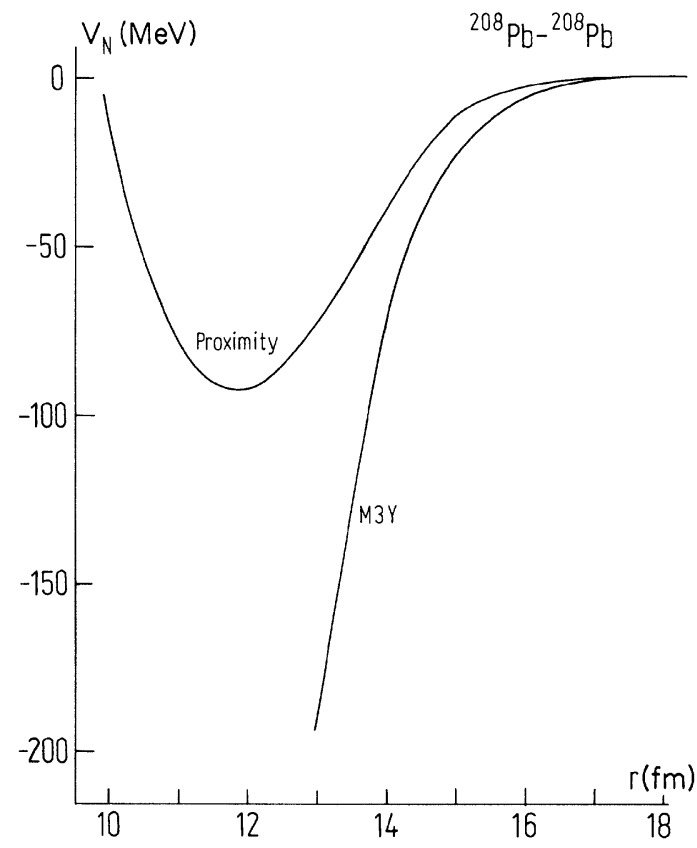

FIG. 10. Comparison of the nuclear potential calculated with the proximity method using a Seyler-Blanchard $\mathrm{N}-\mathrm{N}$ interaction on one hand to the result of the double-folding integral using the M3Y force.

racy of about $10^{-4}$. (For details see Ref. 6.) We have evaluated the proximity potential for two deformed nuclei in complete analogy with the principles and methods of the original paper, ${ }^{2}$ including the special choice of $\phi(s)$. It is interesting to see how the results are modified considering deformation effects. Therefore, we study the nuclear potential of two ${ }^{238} \mathrm{U}$ nuclei as a function of the orientation angles $\beta_{i}$ and the two-center distance $r$. The result is displayed in Fig. 7, where we use the deformation parameters $\delta_{2}=0.264, \delta_{4}=0.106,{ }^{7}$ and $R_{0}=7.44 \mathrm{fm}$. Remarkable minima occur for orientations $\beta_{1}=\beta_{2}=55^{\circ}$ and $\beta_{1}=115^{\circ}, \beta_{2}=65^{\circ}$.

For spherical nuclei in this mass region, minima in the nuclear potential are not observed. Special geometries of the nuclear surface seem to amplify the attractive nuclear force.

The U nucleus has flat surface areas due to its hexadecupole deformation. In such orientations, where two flat areas face each other, the number of nucleons which come into nuclear contact is considerably increased compared to the situation of two curved surfaces. (See Fig. 8.) Clearly these types of nuclear "cohesion". minima, due to facing flat surfaces, are strongly dependent on the hexadecupole deformation. Figure 9 shows the potential using various experimental data of deformation parameters. However, these results differ drastically from those of a double folding calculation ${ }^{6}$ using the M3Y interaction of Love and Satchler. ${ }^{11}$ We now analyze the reasons for these deviations. The proximity description consists of a new principal idea [the proximity theorem: $V_{N}=F($ geometry) $\phi(s)$ ] and a more technical prescription [the calculation of $\phi(s)$ using the Thomas-Fermi method with the Seyler- 

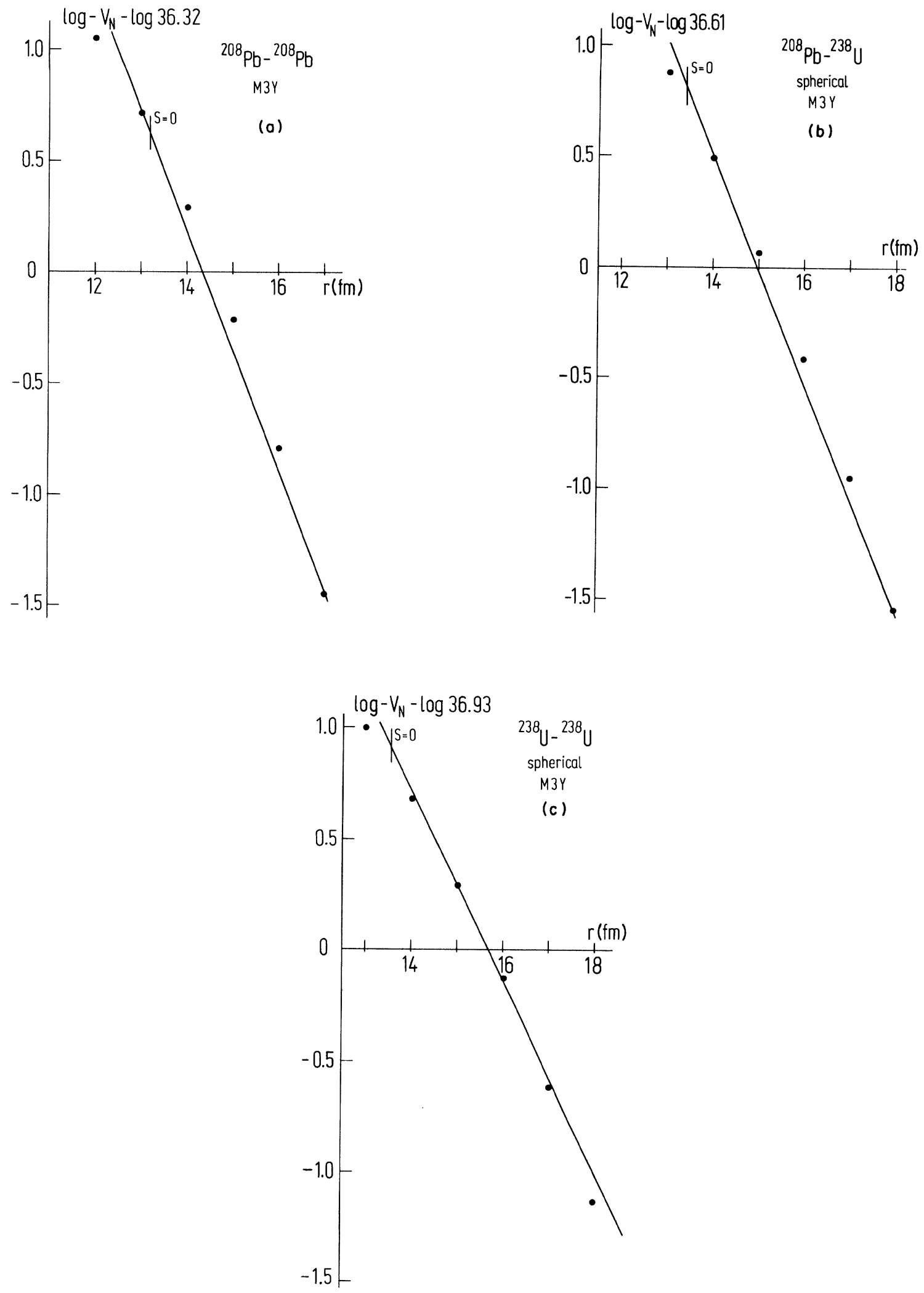

FIG. 11. Fit of the proximity-function $\widetilde{\phi}(s)$ (straight line) to the double folding method (dots) for the systems ${ }^{208} \mathrm{~Pb}-{ }^{208} \mathrm{~Pb}$, ${ }^{208} \mathrm{~Pb}^{238} \mathrm{U}$, and ${ }^{238} \mathrm{U}-{ }^{238} \mathrm{U}$. The $\mathrm{U}$ nucleus is assumed to be spherical in these fits. 

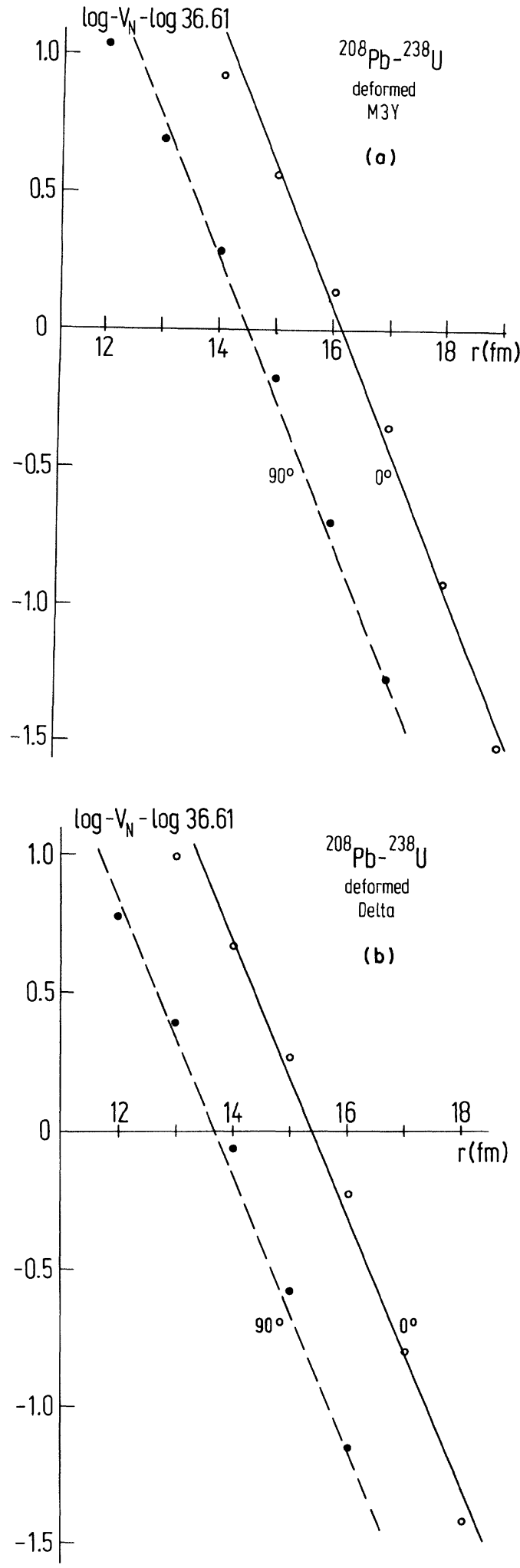

FIG. 12. Comparison between the double-folding integral (dots) and the proximity method (straight lines) fitted in Fig. 11. We show two orientations of the deformed $U$ nucleus: $\beta=0^{\circ}$ (solid line) and $\beta=90^{\circ}$ (dashed line). Two nucleon-nucleon interactions were utilized, the M3Y force (a) and the delta force (b).
Blanchard N-N interaction.]

In order to clarify to what extent the deviations between the double-folding and the proximity potentials are caused by the proximity theorem and by the technical method involved, we first calculate the nuclear potential for two spherical systems (e.g., ${ }^{208} \mathrm{~Pb}-{ }^{208} \mathrm{~Pb}$ ) and compare the result with the double folding method. We use the half-density radius $R_{0}=6.6 \mathrm{fm}$ and the density distribution measured by Heisenberg et al. ${ }^{12}$ for ${ }^{208} \mathrm{~Pb}$

$$
\rho(r)=\frac{\rho_{0}}{1+\exp \left[\left(r^{2}-c^{2}\right) \mid z^{2}\right]}\left(1+w r^{2} / c^{2}\right),
$$

with $c=6.3032 \mathrm{fm}, z=2.8882 \mathrm{fm}$, and $w=0.3379$. The result is displayed in Fig. 10. Apparently, the two models yield quite different results. The proximity potential with the Seyler-Blanchard interaction, Eq. (14), takes the compression energy into account (sudden potential), and therefore increases at negative $s$ values. On the other hand, the double-folding potential with the M3Y force continues to decrease, because compression effects are not considered (adiabatic potential). We conclude that the deviation between the two models is caused to a large extent by different assumptions about the effective $\mathrm{N}-\mathrm{N}$ interaction itself. In addition, the Thomas-Fermi approximation is expected to give rise to some differences. Physically, the fact that the potentials differ is well understandable, because the proximity potential is constructed with respect to an application to fusion cross sections, whereas the double folding model in the present form must be regarded as a fit to elastic scattering. The corresponding nuclear potentials are not expected to agree everywhere. Fusion may occur following direct transitions to nonelastic channels as well as from the entrance channel itself. Consequently, we expect the two potentials to deviate when the nuclei come into contact. For a test of the proximity theorem, we must ensure that the proximity function $\phi(s)$ is based on the same N-N interaction as used in the double folding model. Therefore, we modify the proximity function $\phi(s)$ in such a way that the double-folding potential is reproduced for spherical nuclei. In analogy to the Bass potential, ${ }^{1}$ we write the new function $\widetilde{\phi}(s)$ in the form

$$
\widetilde{\phi}(s)=w e^{-s / a} \text {. }
$$

The parameters $w$ and $a$ are fitted to the M3Y calculation. This is shown in Fig. 11 for $\mathrm{Pb}$ on $\mathrm{Pb}$ using for simplicity a logarithmic scale (Bass potentials are straight lines). The parameters are easily determined:

$$
\begin{aligned}
\log \left(-V_{N}\right) & =\log 4 \pi \gamma \bar{R}+\log (-\widetilde{\phi}(s)) \\
& =\log 36.32+\log \left(-w e^{-s / a}\right), \\
\log \left(-V_{N}\right) & -\log 36.32=\log (-w)-s / a \log e,
\end{aligned}
$$

with

$$
R=\frac{R_{0}^{2}}{2 R_{0}}=3.3 \mathrm{fm},
$$

and the shorthand notation

$$
\log \left(-V_{N}\right) \equiv \log \left(-\frac{V_{N}}{V_{0}}\right)
$$



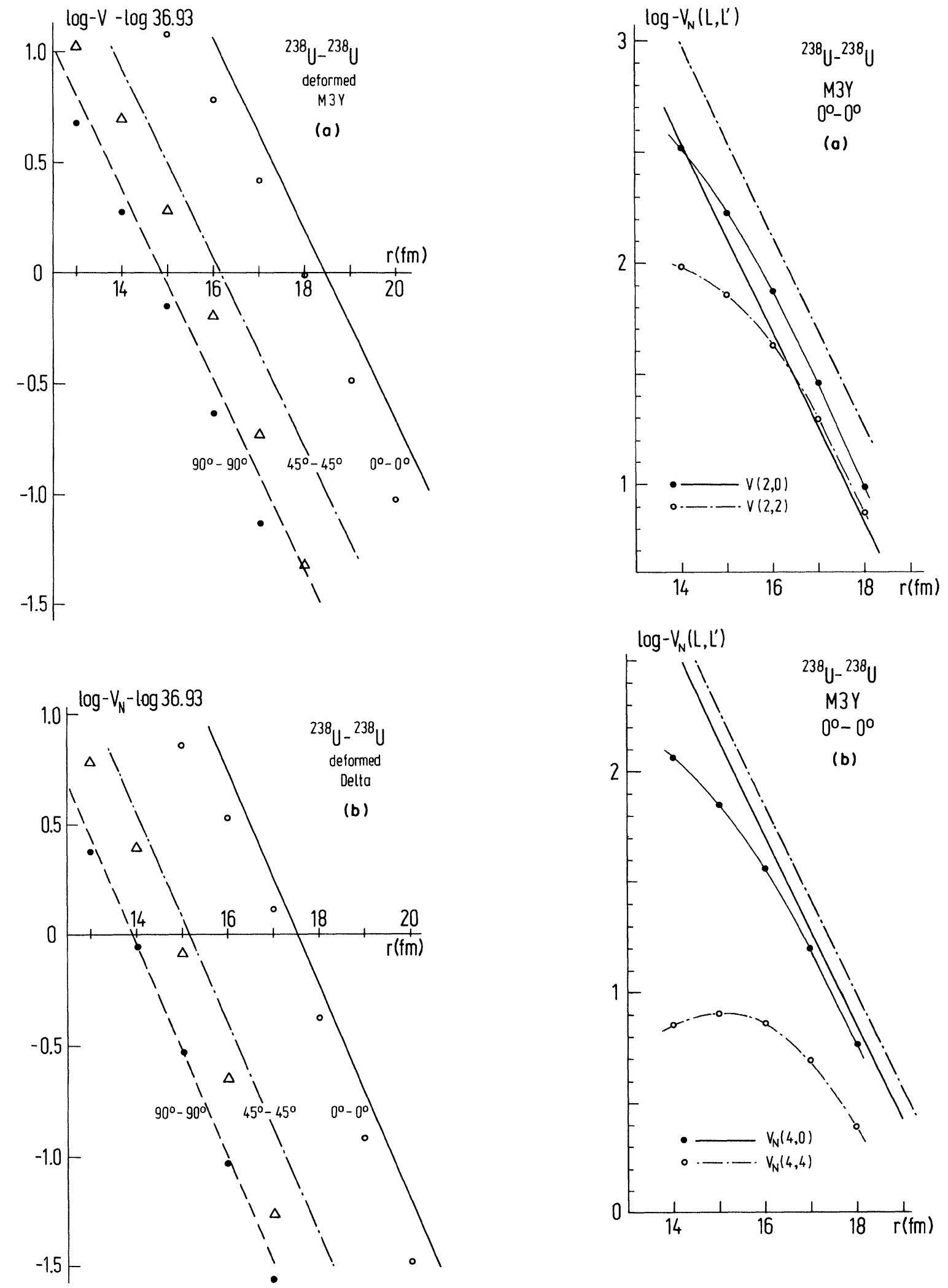

FIG. 13. Comparison as in Fig. 12, but for the double-U system. We show three orientations: $\beta_{1}=\beta_{2}=0^{\circ}$, solid line and open circles; $\beta_{1}=\beta_{2}=45^{\circ}$, dashed-dotted line and triangles; and $\beta_{1}=\beta_{2}=90^{\circ}$, dashed line and full circles.

FIG. 14. Comparison of different multipole components for the system ${ }^{238} U{ }^{238} U$ using the proximity method (straight lines) and the double-folding integral (dots). 
with $V_{0}=1 \mathrm{MeV}$. With the choice of $\widetilde{\phi}(s)$, the models are nearly equivalent for spherical systems. As a next step, we consider the spherical + deformed system $\mathrm{Pb}$-U. For the $U$ nucleus, we use the following density and shape parametrization ${ }^{10}$ :

$$
\begin{aligned}
& \rho(r, \vartheta)=\frac{\rho_{0}}{1+\exp \{[r-R(\vartheta)] / c\}}, \\
& R(\vartheta)=R_{0}\left(1+\delta_{2} Y_{20}+\delta_{4} Y_{40}\right), \\
& R_{0}=6.8054 \mathrm{fm}, \delta_{2}=0.261, \\
& \delta_{4}=0.087, \quad c=0.6049 \mathrm{fm} .
\end{aligned}
$$

The proximity function $\widetilde{\phi}(s)$ for this system has been fitted to the $L=0$ component of the M3Y double folding potential, i.e.,

$$
U\left(r, \beta_{2}\right)=\sum_{L} U_{L}(r) Y_{L 0}\left(\beta_{2}\right)
$$

The two models are compared for the spherical + deformed system $\mathrm{Pb}+\mathrm{U}$ in Fig. 12. We find excellent agreement for all orientation angles. We observe that the potentials for different orientations have the same slope, which proves the validity of the proximity theorem in this case because

$$
\begin{aligned}
\log (-F(\text { geometry }) \phi(s))= & \log (F(\text { geometry })) \\
& +\log (-\phi(s)) .
\end{aligned}
$$

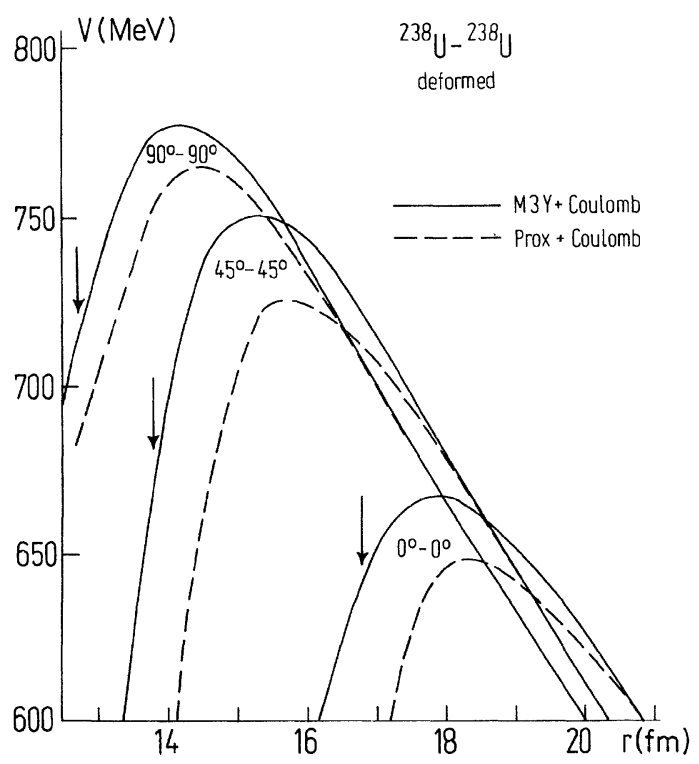

FIG. 15. Comparison of the nuclear and Coulomb potentials in a linear scale. The arrows indicate the surface touching point, i.e., the Fermi densities of both nuclei add to the normal equilibrium density. Solid lines correspond to the double folding method, dashed lines to the proximity potential. We show three different orientations of the deformed nuclei, $\beta_{1}=\beta_{2}=0^{\circ}$, $\beta_{1}=\beta_{2}=45^{\circ}$, and $\beta_{1}=\beta_{2}=90^{\circ}$.
Furthermore, we calculated the double folding potential using a delta function for the N-N interaction. The fit of $\widetilde{\phi}(s)$ has been determined with the same method, leading to the results displayed on the right-hand side of Fig. 12. Also in this case, we find excellent agreement between the double-folding and the proximity methods. As an example for two deformed nuclei, we study the U-U system. The function $\widetilde{\phi}(s)$ is fitted to the monopole-monopole component of the double-folding result using the expansion

$$
\begin{gathered}
U\left(r, \beta_{1}, \beta_{2}\right)=\sum_{L L^{\prime} M} U_{L L^{\prime} M}(r) Y_{L M}\left(\beta_{1}, \varphi=0\right) \\
\times Y_{L^{\prime} M}\left(\beta_{2}, \varphi=0\right)
\end{gathered}
$$

corresponding to Eq. 15 of Ref. 6, i.e., the potential of two spherical $U$ nuclei. The comparison leads to remarkable deviations for both types of interactions (Fig. 13). The proximity method has a tendency to underestimate the nuclear potential; the strongest deviations occur for the orientation $\beta_{1}=\beta_{2}=45^{\circ}$. The analysis of the different multipole components (see Fig. 14) shows that the deviations are mainly caused by the multipole-multipole components. This corresponds to the fact that we find reasonable agreement for systems with one spherical and one deformed nucleus, where the multipole-multipole components vanish. The nuclear plus Coulomb potential is plotted in Fig. 15. The qualitative behavior of both models is the same for all orientations.

The Coulomb barriers of the proximity potential are about $20 \mathrm{MeV}$ lower than in the M3Y calculation. In contrast to the results shown in Figs. 7 and 9, the most favored orientation to overcome the Coulomb barrier is now $\beta_{1}=\beta_{2}=0^{\circ}$. The reason for this change is the different proximity function $\widetilde{\phi}(s)$. The increase of the nuclear attraction due to flat surfaces facing each other is visible also in this calculation. The decrease of the potential taken at the surface touching point (arrows) is strongest for the orientation $\beta_{1}=\beta_{2}=45^{\circ}$. The conclusions about a possible pocket in the ${ }^{238} \mathrm{U}_{-}{ }^{238} \mathrm{U}$ potential ${ }^{6}$ remain unchanged using the proximity method.

\section{SUMMARY AND OUTLOOK}

In the last section, we have shown that the proximity method yields results which are quite similar to those obtained with the double-folding method provided that at least one of the nuclei has a spherical shape and that the same nucleon-nucleon interaction is utilized in both approaches. Baltz and Bayman ${ }^{4}$ arrived at a similar conclusion considering a $\delta$ force. They also investigated an improved calculation of the geometrical factor $F$ in Eq. (1) ("two term proximity model") as suggested by Brink et $a l .{ }^{13}$; this extended version of the proximity model gives rise to an even better agreement with the doublefolding theory, but the deviations in the case of two deformed nuclei remain. In applying the proximity potential to the ${ }^{238} U_{-}{ }^{238} U$ system, we find the remarkable result 
that the nuclear interaction potential is changed dramatically for certain geometrical conditions, e.g., for flat surface areas, due to a strong hexadecupole deformation. In this context, certain "orientation windows" of the nuclei play an important role. Hence, in calculating the nucleus-nucleus potential, it is important that we include higher multipole moments $(l=2,4, \ldots)$ of the density distribution and that we do not simply start from a potential averaged over all orientations.

\section{ACKNOWLEDGMENTS}

We gratefully acknowledge fruitful and stimulating discussions with Professor Rainer Bass. This research was sponsored by the Bundesministerium für Forschung und Technologie and the Gesellschaft für Schwerionenforschung, and by the Division of Basic Energy Sciences, U. S. Department of Energy, under Contract W-7405eng-26 with the Union Carbide Corporation.
${ }^{1}$ R. Bass, Phys. Lett. B47, 139 (1973).

2J. Blocki, J. Randrup, W. Swiatecki, and C. F. Tsang, Ann. Phys. (N.Y.) 105, 427 (1977).

3J. Randrup and J. S. Vaagen, Phys. Lett. B77, 170 (1978).

${ }^{4}$ A. J. Baltz and B. F. Bayman, Phys. Rev. C 26, 1969 (1982).

${ }^{5}$ R. G. Seyler and C. H. Blanchard, Phys. Rev. 124, 227 (1961); 131,355 (1963).

6M. J. Rhoades-Brown, V. E. Oberacker, M. Seiwert, and W. Greiner, Z. Phys. A $\underline{310}, 287$ (1983).

${ }^{7}$ F. K. McGowan, C. E. Bemis, Jr., J. L. C. Ford, Jr., W. T. Milner, R. L. Robinson, and P. H. Stelson, Phys. Rev. Lett.
27, 1741 (1971).

8J. P. Davidson, D. A. Close, and J. J. Malanify, Phys. Rev. Lett. 32,337 (1974).

${ }^{9}$ R. M. Ronningen, R. C. Melin, J. A. Nolen, Jr., G. M. Crawley, and C. E. Bemis, Jr., Phys. Rev. Lett. 47, 635 (1981).

${ }^{10}$ T. Cooper, W. Bertozzi, J. Heisenberg, S. Kowalski, W. Turchinetz, C. Williamson, L. Cardman, S. Fivozinsky, J. Lightbody, Jr., and S. Penner, Phys. Rev. C 13, 1083 (1976).

${ }^{11}$ G. R. Satchler and W. G. Love, Phys. Rep. 55, 183 (1979).

12J. H. Heisenberg and I. Sick, Phys. Lett. 32B, 249 (1970).

13D. M. Brink and Fl. Stancu, Nucl. Phys. A299, 321 (1978). 